\title{
SÉRIE TEMPORAL DOS ÓBITOS DE CASOS INTERNADOS POR COVID-I9 ENTRE 2020 E 202I NA FHEMIG
}

\author{
Deise Campos Cardoso \\ deise.campos@fhemig.mg.gov.br \\ Fabiana Guerra Pimenta \\ Fundação Hospitalar do Estado de Minas Gerais/ FHEMIG \\ fabiana.pimenta@fhemig.mg.gov.br \\ Luiza Mara Moura Tameirão \\ luizammt@hotmail.com \\ Maria Thereza Coelho Papatela Jabour \\ maria.jabour@fhemig.mg.gov.br
}

\section{RESUMO}

INTRODUÇÃO: A pandemia por COVID-I9 se manifestou por flutuações no quantitativo de casos e óbitos que foram denominadas "ondas". Espera-se um efeito do início da vacinação em Minas Gerais e seus efeitos sobre as "ondas" no estado e dos óbitos de pacientes internados. OBJETIVO: Verificar a tendência temporal de casos internados por COVID-19 que vieram a óbito na FHEMIG em 2020 e 202I. METODOLOGIA: Dados quinzenais enviados pelas Unidades Assistenciais entre as Semanas Epidemiológicas 34-2020 a 26-202I foram consolidados para análise. Para o ano de 202I, incluiu-se a Semana Epidemiológica 53 de 2020. Utilizaram-se linhas de tendência do Microsoft Excel (2016). Participaram os Hospitais Eduardo de Menezes, Júlia Kubitschek, Infantil João Paulo II, João XXIII, Regional de Barbacena, Regional João Penido e Regional Antônio Dias. APROVAÇÃO DO CEP: Parecer Consubstanciado do CEP N4637284, de 08 de abril de 202I. RESULTADOS: Verificou-se uma média móvel de número de casos internados por COVID-I9 na FHEMIG maior a partir de março de 202I em relação a 2020. Não houve diferença na relação por sexo dentre os óbitos no período estudado. Em 2020, a proporção de óbitos em pessoas com 60 anos e mais manteve-se constante, mas observou-se queda em 202I ( $\left.\mathrm{R}^{2}=0,5965\right)$. A proporção quinzenal de óbitos em pessoas sem comorbidades aumentou no período 2020 a $202 \mathrm{I}\left(\mathrm{R}^{2}=0,7420\right)$. A proporção de óbitos em pessoas residentes em município diferente da sede foi menor que no ano de 2020 entre os meses de março e maio de $202 \mathrm{I}$ (medianas 68,86 e 65,05, respectivamente). As taxas de letalidade se mantiveram constante em 2020. Em 202I, verificou-se queda na taxa de letalidade hospitalar e por utilização de $\mathrm{VM}$, e aumento na taxa de letalidade por internação em CTI $\left(\mathrm{R}^{2}=0,5942, \mathrm{R}^{2}=0,654 \mathrm{I}\right.$ e $\mathrm{R}^{2}=0,6666$, respectivamente). CONCLUSÃO: Nos hospitais da FHEMIG onde ocorreram óbitos por COVID-19, observou-se alteração no comportamento dos dados quando se comparou 2020 e 202I. Em especial, verificou-se aumento na proporção de óbitos sem comorbidades e diminuição dentre os pacientes de 60 anos e mais, possivelmente pela maior gravidade dos casos internados e pela introdução da vacina no início de 2021 .

PALAVRAS CHAVES: Epidemiologia dos serviços de saúde, Síndrome Respiratório Aguda Grave, Pandemias por COVID-I9, Séries Temporais. 